\title{
The Prevalence of Cryptosporidium Infection Among Renal Transplanted Patients in Hamadan City, West of Iran
}

\author{
Rasool Jafari ${ }^{1}$; Zahra Gharibi ${ }^{2}$; Mohammad Fallah ${ }^{2, *}$ \\ ${ }^{1}$ Department of Parasitology and Mycology, School of Medicine, Isfahan University of Medical Sciences, Isfahan, IR Iran \\ ${ }^{2}$ Department of Parasitology, School of Medicine, Hamadan University of Medical Sciences, Hamadan, IR Iran \\ *Corresponding author: Mohammad Fallah, Deptartment of Parasitology, School of Medicine, Hamadan University of Medical Sciences, Hamadan, IR Iran. Tel: +98-9181113650, Fax: \\ +98-8112520203, E-mail: mohfall@yahoo.com, fallah@umsha.ac.ir
}

Received: January 6, 2014; Revised: February 15, 2014; Accepted: March 13, 2014

\begin{abstract}
Background: Opportunistic infections have become much more considerable in the last decades, especially in immunocompromised patients and due to the medical interventions. Cryptosporidium is a pathogenic protozoan parasite causing diarrhea in children and some times acts as a life threatening opportunistic pathogen in the immunocompromised adults.

Objectives: This study aimed to investigate the presence of Cryptosporidium infection among patients undergone renal transplantation, who are at risk for this infection.

Patients and Methods: This was a cross-sectional study and the sample collection consisted of 180 renal transplanted patients referred to Shaheed-Beheshti Hospital, Hamadan city, Iran. The stool specimens were concentrated using formalin-ether technique and then the fecal smears were prepared from the sediments. Afterwards, the slides were stained using the Ziehl-Neelsen staining method and then examined for the presence of Cryptosporidium oocysts.

Results: One out of 180 fecal samples was positive for Cryptosporidium infection. The infected patient was a 51-year-old woman who had a renal transplantation six years earlier, with continuous use of CellCept ${ }^{\circledR}$ (mycophenolate mofetil) and prednisolone. The patient had been referred to the hospital with gastrointestinal symptoms.

Conclusions: Based on the results of this study the prevalence of cryptosporidiosis was very low in renal transplanted patients in Hamadan city, Iran. It could be concluded that cryptosporidiosis is not a life threatening risk in this region and it probably showed well post-transplantation hygienic status of the patients and/or low oocysts load in the area.
\end{abstract}

Keywords:Cryptosporidium; Immunocompromised Host; Transplants; Iran

\section{Background}

Cryptosporidium is an oocyst-forming protozoan parasite which infects the gastrointestinal tract in human and some other animals. The infection mostly occurs in children and is benign and self-limiting, but could be life threatening in immunocompromised individuals $(1,2)$. It was first described as a cause of gastroenteritis in human in 1976 (1). The parasite has been the cause of some major outbreaks and also sporadic gastroenteritis in developing countries (3). Cryptosporidium is responsible for the one of the greatest documented waterborne outbreaks. The infection mostly results in a benign self-limited watery diarrhea and mostly lasts for days and in some cases, for weeks (4). The major species causing human cryptosporidiosis are C. hominis and C. parvum $(1,5)$. In immunocompromised individuals like HIV positive patients and patients undergone organ transplantation, the infection becomes more invasive resulting in severe diarrhea and sometimes spreads throughout other organs and makes extra-intestinal infection $(2,4,6)$. Drinking water could be an infection source for cryptosporidiosis, therefore the immunocompromised individuals should boil water before drinking it (7). Nowadays by using antiviral drugs against HIV, the HIV-associated opportunistic infections are decreasing, but on the other hand there is an increase in organ transplantation. The organ transplanted patients, as well as other patients with immunodeficiency, are at risk of infection due to the continuous immunosuppressive therapies $(1,8)$.

\section{Objectives}

The organ transplanted patients are at risk of severe cryptosporidiosis and continuous monitoring of the infection among them is necessary. We conducted a crosssectional study which aims to investigate the prevalence of Cryptosporidium infection among renal transplanted patients in Hamadan city, IR Iran.

\section{Patients and Methods}

A total of 180 fecal samples were collected from renal

Implication for health policy/practice/research/medical education: Implication of this article is for health policy and health practice as well.

Copyright @ 2014, Hamadan University of Medical Sciences; Published by Safnek. This is an open-access article distributed under the terms of the Creative Commons Attribution License, which permits unrestricted use, distribution, and reproduction in any medium, provided the original work is properly cited. 
Jafari Ret al.

transplanted patients referred to Shaheed-Beheshti Hospital, Hamadan city, IR Iran, in eight months (winter, spring and summer 2012). Fecal samples were preserved in $10 \%$ formalin and kept in cool and dry place until the examination. Demographic variables of the patients were also collected including: time of transplantation, kind of immunosuppressive drug, age, sex, residential status and gastrointestinal symptoms. Immunorel ${ }^{\circledR}$ (human immunoglobulin), Sandimmune ${ }^{\circledR}$ (cyclosporine), prednisolone and CellCept ${ }^{\circledR}$ (mycophenolate mofetil) were the medications used by patients.

\subsection{Smear Preparation and Staining}

The stool samples were concentrated using formalinether technique. Thin methanol fixed fecal smears were prepared along with positive controls and then stained by cold modified Ziehl-Neelsen staining method (2). The staining method is briefly as explained here : the methanol fixed smears were stained in carbol fuchsin (15 minutes), rinsed in tap water, decolorized with $1 \%$ hydrochloric acid (30 seconds), rinsed in tap water and re-stained with $0.4 \%$ malachite green (30 seconds), again rinsed in tap water and finally dried in air for microscopic examination. Cryptosporidium oocysts are observed as reddish 4-5 $\mu \mathrm{m}$ spherical objects with crescent shaped internal structures (Figure 1) (5).

\subsection{Data Analysis}

Data were analyzed using SPSS software and Chi-square test.

\section{Results}

Out of 180 patients $86(47.7 \%)$ and 94 (52.3\%) were female and male, respectively. The mean age of the participants was 42 (14 to 70) and the average duration of immunosuppressive drug use was 5.5 years. Only a 51-year-old woman from Hamadan city having had renal transplantation six years prior to the study had a positive fecal smear for Cryptosporidium oocysts by Ziehl-Neelsen staining method (0.55\%).

She also had gastrointestinal symptom (diarrhea) and was using CellCept ${ }^{\circledR}$ (mycophenolate mofetil) and prednisolone at the moment. As the prevalence of the infection was very low, the infection showed no statistical relationship to the demographic variables.

\section{Discussion}

In this study, the overall prevalence of cryptosporidiosis was $0.55 \%$, showing very low infection rate and also showing that cryptosporidiosis is present in organ transplanted patients in Hamadan city, west of Iran. Various studies in Iran reported the prevalence rates close to the results of this study (5, 9-11) and some reported data suggest incredibly high infection rates (12). Our findings are

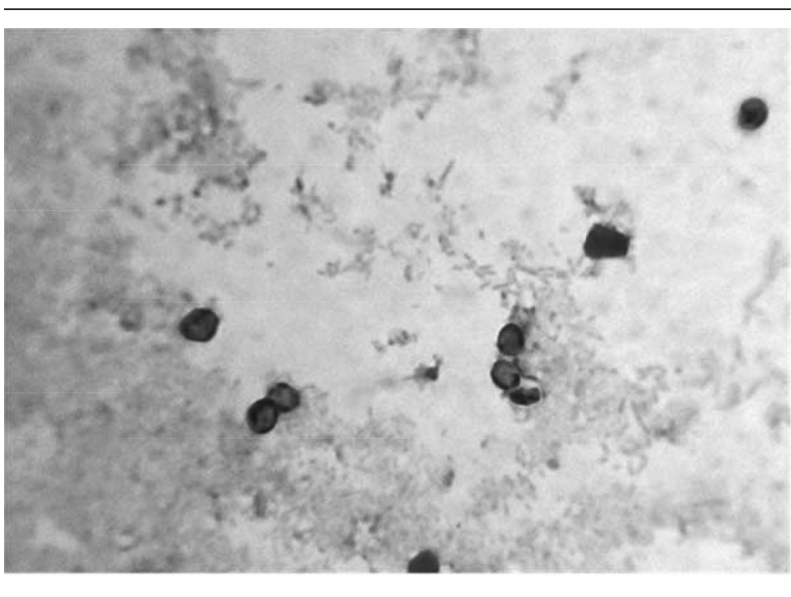

Figure 1. Cryptosporidium Oocysts Observed in a 51-Year-old Renal Transplantation Recipient

aligned with most of the other reports in Hamadan area. For example Heidari et al. reported the infection rate of $1.5 \%$ in asymptomatic individuals from Hamadan city (10), which is very close to our findings. In another study, Jafari et al. reported a $0.87 \%$ infection rate among farmers in the rural area of Hamadan city (5). The prevalence rate of the infection in children with diarrhea was $5.6 \%$ in the city, in 1996, reported by Fallah et al. (13). Comparing the data from Hamadan area with other reports from other parts of the country, we noted that this region carries lower infection load. These findings showed that the general population is not at a considerable risk of infection in this area, which is probably attributable to the immunocompromised patients, as well.

There are similar studies carried out on the prevalence of the infection in solid organ transplanted patients, with the results showing incredibly higher prevalence compared to ours. Some reports of cryptosporidiosis prevalence in organ transplanted patients are as follows: $11.5 \%$ (14), 18.8\% (15), 20\% (16) and 34.8\% (17) in Iran, Turkey, India and Brazil, respectively. Additionally, some researchers in Iran like Sharif et al. (18), Nahravanian et al. (19) and Hazrati Tappeh et al. (14) reported the prevalence of the infection among immunosuppressed patients as $5 \%, 17.3 \%$ and $11.5 \%$, respectively. The studies mentioned above illustrated fairly higher prevalence of the cryptosporidiosis in organ transplanted patients than ours. As recently reported, the drinking water of Hamadan city carries Cryptosporidium oocyst and Giardia cysts (20). It is apparent that oocysts are capable of infecting the immunosuppressed patients, with the lowest dose possible. Therefore the low prevalence found by this study showed a very good hygienic condition in the general population, also attributable to organ transplanted patients. Treatment of cryptosporidiosis and controlling it to avoid extra-intestinal infections is not completely possible, especially in immunocompromised populations. Therefore, preventive methods like boiling the drinking water and not being in 
contact with infected pets, livestock and people seem to be essential. Probably due to the proper observations applied in Hamadan city, the prevalence of the infection is lower compared to the results from other studies, carried out in other regions. Based on the results of this study the prevalence of cryptosporidiosis was very low in renal transplanted patients in Hamadan area, Iran. It could be concluded that cryptosporidiosis is not a life threatening risk in the region. Additionally the findings were demonstrative of a well post-transplantation hygienic status in patients and/or low oocysts load in the area.

\section{Acknowledgements}

The authors would like to thank Vice-chancellor of Research and Technology, Hamadan University of Medical Sciences for the approval of this study and the authorities of Shaheed-Beheshti Hospital, Hamadan, for their contributions.

\section{Authors' Contribution}

The corresponding author was research project advisor and first and second authors were administrators of the project.

\section{Financial Disclosure}

The authors declared no conflict of interests.

\section{Funding/Support}

This study was supported financially by Vice-chancellor of Research and Technology, Hamadan University of Medical Sciences.

\section{References}

1. Shirley DA, Moonah SN, Kotloff KL. Burden of disease from cryptosporidiosis. Curr Opin Infect Dis. 2012;25(5):555-63.

2. Fayer R, Xiao L. Cryptosporidium and cryptosporidiosis. 2nd ed. Boca Raton: CRC Press and IWA Publishing; 2008.

3. Abubakar I, Aliyu SH, Arumugam C, Usman NK, Hunter PR. Treatment of cryptosporidiosis in immunocompromised individuals: systematic review and meta-analysis. Br J Clin Pharmacol. 2007;63(4):387-93.

4. Hunter PR, Nichols G. Epidemiology and clinical features of Cryptosporidium infection in immunocompromised patients. Clin Microbiol Rev. 2002;15(1):145-54.

5. Jafari R, Maghsood AH, Fallah M. Prevalence of Cryptosporidium Infection among Livestock and Humans in Contact with Livestock in Hamadan District, Iran, 2012. J Res Health Sci. 2012;13(1):86-9.

6. Feasey NA, Healey P, Gordon MA. Review article: the aetiology, investigation and management of diarrhoea in the HIV-positive patient. Aliment Pharmacol Ther. 2011;34(6):587-603.

7. Masur H, Kaplan JE, Holmes KK, U. S. Public Health Service. Infectious Diseases Society of America.. Guidelines for preventing opportunistic infections among HIV-infected persons--2002. Recommendations of the U.S. Public Health Service and the Infectious Diseases Society of America. Ann Intern Med. 2002;137(5 Pt 2):435-78.

8. Kaplan JE, Hanson D, Dworkin MS, Frederick T, Bertolli J, Lindegren ML, et al. Epidemiology of human immunodeficiency virus-associated opportunistic infections in the United States in the era of highly active antiretroviral therapy. Clin Infect Dis. 2000;30 Suppl 1:S5-14.

9. Dabir zadeh M, Baghaei M, Bakaiyan M, Ghudarzi M. [Prevalence of cryptosporidium in Diarrheal children under 5 years old admitted to Pediatric hospital in Zahedan City, Iran].J Gorgan Univ Med Sci. 2003;11(5):54-9.

10. Heidari, H, Gharakhani J. Study of Cryptosporidium Infection in the Livestock (Cattle, Sheep, Dogs, Fowls) and Humans, in Hamadan City and Its Suburbs during 2006-2011. Sci J Hamadan Univ Med Sci. 2012;19(3):67-74.

11. Kia EB, Hosseini M, Nilforoushan MR, Meamar AR, Rezaeian M. Study of intestinal protozoan parasites in rural inhabitants of Mazandaran province, Northern Iran. Iran J Parasitol. 2008;3(1).

12. Sazmand A, Rasooli A, Nouri M, Hamidinejat H, Hekmatimoghaddam S. Prevalence of Cryptosporidium spp. in Camels and Involved People in Yazd Province, Iran. Iran J Parasitol. 2012;7(1):80-4.

13. Fallah M, Haghighi A. Cryptosporidiosis in children with diarrhea submitted to Health centers in the west of Iran (Hamadan). Med J Islamic Rep Iran. 1996;9(4):315-7.

14. Hazrati Tappeh KH, Gharavi MJ, Makhdoumi K, Rahbar M, Taghizadeh A. Prevalence of Cryptosporidium spp. Infection in Renal Transplant and Hemodialysis Patients. Iran J Public Health. 2006;35(3):54-7.

15. Ok UZ, Cirit M, Uner A, Ok E, Akcicek F, Basci A, et al. Cryptosporidiosis and blastocystosis in renal transplant recipients. Nephron. 1997;75(2):171-4.

16. Udgiri N, Minz M, Kashyap R, Heer M, Gupta CS, Mohandas K, et al. Intestinal cryptosporidiasis in living related renal transplant recipients. Transplant Proc. 2004;36(7):2128-9.

17. Chieffi PP, Sens YA, Paschoalotti MA, Miorin LA, Silva HG, Jabur P. Infection by Cryptosporidium parvum in renal patients submitted to renal transplant or hemodialysis. Rev Soc Bras Med Trop.1998;31(4):333-7.

18. Sharif M, Ziyaei-Hezar-Jaribi H, Gholami S. [Prevalence of cryptosporidiosis in patients using immunosupressive drugs]. $J$ Guilan Univ Med Sci. 2006;13(51):16-22.

19. Nahrevanian H, Assmar M, Ghorbani SM. A Study on Cryptosporidiosis Among Patients With Acquired Immunodeficiency in The Great Tehran. J School Pub Health Inst Pub Health Res. 2005;3(2):77-86.

20. Fallah M, Bastaminejad S, Maghsood AH, Rahmani A, Kakaee $\mathrm{H}$, Akbari A. [Detection of Giardia Cysts and Cryptosporidium Oocysts in Drinking Water Rresources in Hamadan City]. J Ilam Univ Med Sci. 2013;21(5):29-33. 